\title{
Nontraumatic Cervical Internal Carotid Artery Pseudoaneurysm in a 16-Month-Old Child Treated by Coil Embolization
}

\author{
K. Nagarajan ${ }^{1}$ () \\ K. Ariharan ${ }^{1 \odot}$ \\ D. Sunilkumar ${ }^{1 \odot}$ \\ ${ }^{1}$ Department of Radio-Diagnosis, Jawaharlal Institute of Postgraduate \\ Medical Education \& Research, JIPMER, Pondicherry, India \\ 2 Deparment of Paediatrics, Jawaharlal Institute of Postgraduate \\ Medical Education \& Research, JIPMER, Pondicherry, India
}

J Clin Interv Radiol ISVIR 2022;6:226-228.

\author{
Abraar Sheriff 20
}

\author{
Address for correspondence K. Nagarajan, MD RD, DM \\ Neuroradiology Professor, Department of Radio-Diagnosis, Jawaharlal \\ Institute of Postgraduate Medical Education \& Research, JIPMER, \\ Pondicherry 605006, India (e-mail: Iknagarajan1@gmail.com).
}

A 16-month-old female child presented with a single episode of epistaxis $(\sim 100 \mathrm{~mL})$ and intermittent high-grade fever. The child also had a single episode of blood-stained vomitus the previous day. No history of trauma or other bleeding manifestations was present. Clinical examination revealed severe pallor, tachycardia, and hypertension (104/70 mm $\mathrm{Hg}$ ). Local examination of the nasal cavity and pharynx showed no obvious pathology. The epistaxis was considered secondary to hypertension. Further episodes of epistaxis in the hospital were managed with anterior and posterior nasal packing. The child developed hypotension and was stabilized with intravenous fluids and packed red cell transfusion. Computed tomography angiogram (CTA) revealed a large pseudoaneurysm $(24 \times 14 \mathrm{~mm})$ from the cervical segment of the left internal carotid artery (ICA) with hematoma in the left parapharyngeal space (-Fig. 1). There was no contrast opacification of distal ICA beyond the pseudoaneurysm. There were no infarcts in bilateral cerebral hemispheres. After informed consent from the parents, digital subtraction angiography (DSA) was done under sedation using left common femoral artery access and 4F diagnostic catheter. DSA of the left ICA showed a slow filling pseudoaneurysm with a narrow neck from the left cervical ICA. No flow was noted in the left ICA distal to the pseudoaneurysm. Right ICA injection showed normal filling of left middle cerebral artery and anterior cerebral artery via the Circle of Willis (-Fig. 2). Coil embolization of the pseudoaneurysm neck and adjacent parent left ICA was done using coils (Nester, $4 \times 14 \mathrm{~mm}$ ). Postprocedure check angiogram revealed complete occlusion of the pseudoaneurysm neck and left ICA. The procedure was uneventful. The next day, nasal packing was removed and there were no further episodes of epistaxis. She had persistent hypertension that was initially managed by sodium nitroprusside infusion and gradually changed over to oral amlodipine. On follow-up, she had mild right hemiparesis that improved in 3 months of almost 5/5 power. Due to her hypertension, vasculitis workup was done, but it turned out to be negative.

Extracranial ICA pseudoaneurysms are rare in children.

They are usually a complication of deep neck space infection but can also be caused by trauma, vasculitis, and iatrogenic procedures. ${ }^{1}$ These patients can deteriorate rapidly if there is bleeding into the nasal cavity or pharynx. The youngest previously reported case of ICA pseudoaneurysm coiling was by Roos and Butler in a 2-year-old child. ${ }^{2}$

The earlier reports of cervical internal carotid pseudoaneurysms in pediatric age group were due to infections including tonsillitis, impaction of food material (like fishbone), vasculitis, collagen vascular disorders, and iatrogenic procedures apart from trauma. ${ }^{3-6}$

Singhal et $\mathrm{al}^{7}$ have reported two cases of ICA pseudoaneurysm formation in an 8- and 2-year-old children after fine-needle aspiration of cervical lymph nodes. Jean Roger et $\mathrm{al}^{8}$ and Karim et $\mathrm{al}^{9}$ have reported pseudoaneurysm formation in the common carotid arteries following fishbone impaction. Both these cases underwent surgical repair with good outcome. Sundarrajan et al $^{10}$ recently reviewed the treatment of large infectious extracranial carotid artery pseudoaneurysms in children and found 26 patients. This included 17 males (65.4\%) and the median age of
DOI https://doi.org/ 10.1055/s-0041-1742231. ISSN 2457-0214. (c) 2022. Indian Society of Vascular and Interventional Radiology. All rights reserved.

This is an open access article published by Thieme under the terms of the Creative Commons Attribution-NonDerivative-NonCommercial-License, permitting copying and reproduction so long as the original work is given appropriate credit. Contents may not be used for commercial purposes, or adapted, remixed, transformed or built upon. (https://creativecommons.org/ licenses/by-nc-nd/4.0/)

Thieme Medical and Scientific Publishers Pvt. Ltd., A-12, 2nd Floor, Sector 2, Noida-201301 UP, India 


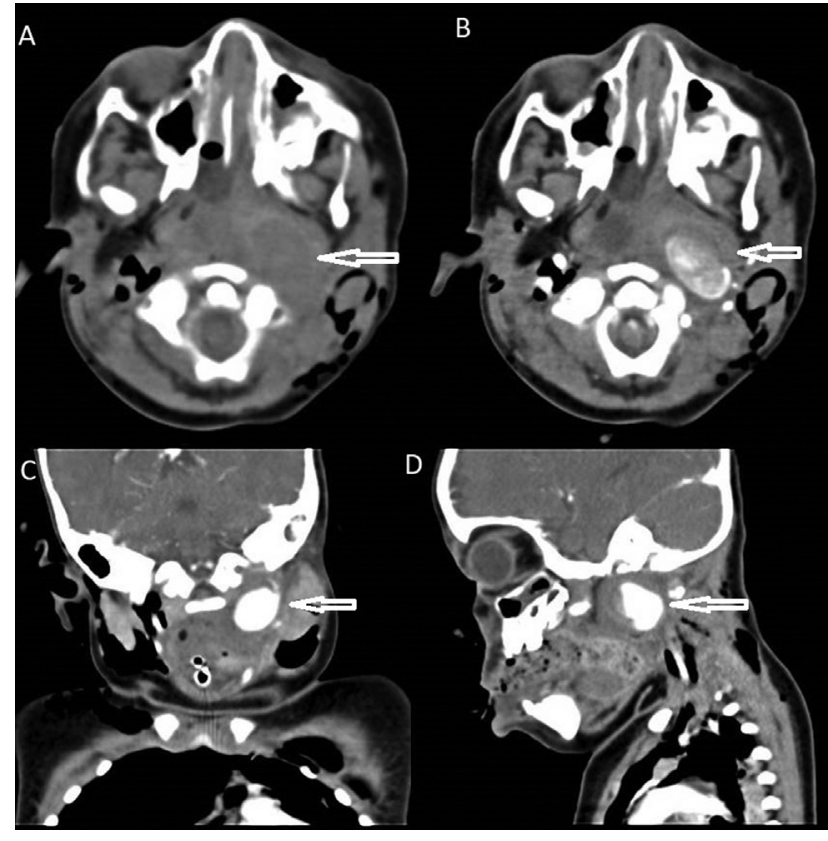

Fig. 1 Plain computed tomography (CT) axial section (A) showing left parapharyngeal soft issue density lesion with peripheral hyperdensity. $\mathrm{CT}$ angiography axial section (B), coronal (C), and sagittal reformations (D) showing arterial-phase enhancement of the left parapharyngeal lesion in continuity with left internal carotid artery suggestive of pseudoaneurysm (arrows).

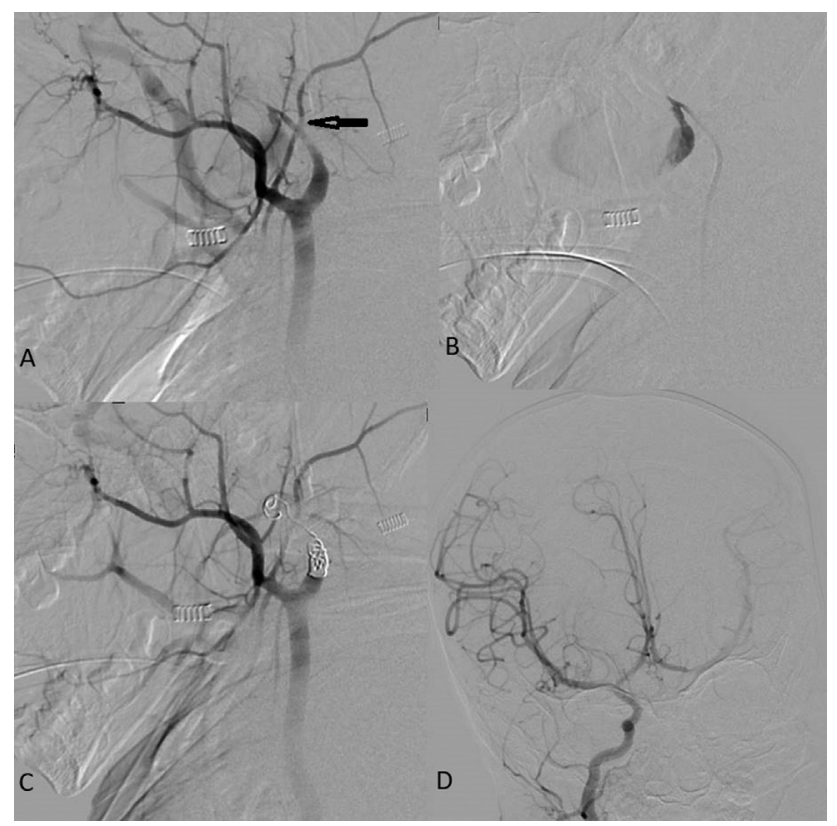

Fig. 2 Digital subtraction angiography of left common carotid artery showing tapering stump of internal carotid artery opening into pseudoaneurysmal sac (arrow in A). Selective microcatheter injection at the neck of the pseudoaneurysm (B). No distal flow intracranially noted. Postcoil deployment shows exclusion of the aneurysm (C). Right internal carotid artery injection showing filling of the left anterior cerebral artery and middle cerebral artery (D).

presentation was 4 years. In this review, 23 (88.5\%) pseudoaneurysms were on the ICA and $2(7.7 \%)$ were on the external carotid artery. One pseudoaneurysm was located on the common carotid artery. The most common presentation was hemorrhage that occurred in eight patients (30.8\%) and the most common causative organism was Streptococcus $s p$. (9 cases out of 15 cases with pathogen report). Of the 18 patients treated with endovascular technique, 14 underwent coil embolization of the parent vessel, 3 underwent carotid stenting, and 1 underwent vascular plug deployment. Reported postprocedural neurological deficits include facial nerve palsy, vocal cord paralysis, Horner's syndrome, and hemiparesis.

In their review of eight cases of pediatric neck arterial pseudoaneurysms, Balasundaram et $\mathrm{al}^{1}$ have suggested a management protocol based on presentation and collateral flow.

In emergency conditions with a good intracranial collateral flow, parent vessel occlusion can be done.

Previous reports also mention about watershed infarct in the ipsilateral hemisphere with some functional impairment after endovascular coil embolization of parent ICA for pseudoaneurysm, though the intracranial collateral flow was angiographically adequate. ${ }^{11,12}$ The child in our report developed mild right hemiparesis postprocedure at 1 week. If the collateral circulation is not adequate, then the parent artery may be preserved by stent-graft or surgical ligation of pseudoaneurysm and end-to-end anastomosis. Coil embolization into the pseudoaneurysm sac is not preferred as it may erode into the mucosa and bleed profusely. Other novel methods used in treatment of these pseudoaneurysms include using $n$-butyl cyanoacrylate (glue) in a case of spontaneous ICA pseudoaneurysm in a 3 -year-old child. ${ }^{13}$ Intraaneurysmal thrombin injection is usually not done in carotid pseudoaneurysms due to the chances of recurrence and distal embolization. Complications following endovascular management include coil migration, recanalization of the pseudoaneurysm, and embolic stroke. The data on the long-term follow-up and outcome is limited due to fewer number of reported cases. The etiology of the pseudoaneurysm in our case was not clear and though the child's hypertension was considered due to vasculitis, the workup was negative.

Conflict of Interest

None declared.

\section{References}

1 Balasundaram P, Sebastian LJD, Jain N, Prabhakar A, Garg A, Gaikwad S. Management of arterial pseudoaneurysms of the neck in a pediatric population: an endovascular case series and review of literature. World Neurosurg 2019;125:e273-e281

2 Roos M, Butler I. Extracranial internal carotid artery pseudoaneurysm in a two-year-old child: case report. J Laryngol Otol 2016;130(06):596-599

3 Watson MG, Robertson AS, Colquhoun IR. Pseudoaneurysm of the internal carotid artery: a forgotten complication of tonsillitis? J Laryngol Otol 1991;105(07):588-590

4 Bersani D, Lanchier C, Lippa A, Saint-Martin J, Choulot JJ. [Pseudoaneurysm of the internal carotid artery]. J Radiol 1992;73(02): $115-119$

5 Krysl J, Noël de Tilly L, Armstrong D. Pseudoaneurysm of the internal carotid artery: complication of deep neck space infection. AJNR Am J Neuroradiol 1993;14(03):696-698 
6 Reisner A, Marshall GS, Bryant K, Postel GC, Eberly SM. Endovascular occlusion of a carotid pseudoaneurysm complicating deep neck space infection in a child. Case report. J Neurosurg 1999;91 (03):510-514

7 Singhal M, Gupta V, Singh P, Lal A, Behra A, Khandelwal N. Iatrogenic life-threatening pseudoaneurysms of extracranial internal carotid artery after fine-needle aspiration of cervical lymph nodes: report of two cases in children. Cardiovasc Intervent Radiol 2009;32(06):1260-1263

8 Jean Roger MT, Marcus F, Emmanuel F, Boniface M, Alain Georges J. Pseudoaneurysm of the common carotid artery in an infant due to swallowed fish bone. Case Rep Radiol 2015;2015:903150. Doi: $10.1155 / 2015 / 903150$

9 Karim HH, Saikia MK, Mitra JK, Medhi J. Impacted fish bone leading to rapidly growing common carotid artery pseudoaneurysm. Indian J Vasc Endovasc Surg 2015;2:35-37
10 Sundarrajan C, Isa SA, Caruso JP, et al. Treatment of large infectious extracranial carotid artery pseudoaneurysms in children: a systematic review of the literature. Childs Nerv Syst 2021;37(05): 1461-1470

11 Brinjikji W, Diehn FE, Lindsay CW, Morris JM. Endovascular treatment of an infected pseudoaneurysm secondary to retropharyngeal abscess in a child. Interv Neuroradiol 2015;21(04): 538-542

12 Ruff MW, Nasr DM, Klaas JP, Renaud DL. Internal carotid artery pseudoaneurysm and ischemic stroke secondary to retropharyngeal and parapharyngeal abscess. J Child Neurol 2017;32(02): 230-236

13 Jagetia A, Sharma D, Singh D, et al. Endovascular occlusion of cervical internal carotid artery pseudoaneurysm in a child treated by n-butyl cyanoacrylate: a rare case report. Pediatr Neurosurg 2015;50(03):168-172 